\title{
Support Vector Machine Detection of Peer-to-Peer Traffic in High-Performance Routers with Packet Sampling: Nonlinear Kernel Approach
}

\author{
F.J. González-Castaño ${ }^{1}$, P.S. Rodríguez-Hernández ${ }^{1}$, R.P. Martínez-Álvarez ${ }^{1}$, \\ and A. Gómez-Tato ${ }^{2}$ \\ ${ }^{1}$ Departamento de Ingeniería Telemática, Univ. de Vigo, Spain \\ ${ }^{2}$ CESGA, Spain \\ \{javier, pedro,rmartinez\}@det.uvigo.es, agomez@cesga.es
}

\begin{abstract}
In this paper, we apply nonlinear support vector machines to identify peer-to-peer ( $\mathrm{p} 2 \mathrm{p})$ traffic in high-performance routers with packet sampling. Due to their high port rates, those routers cannot extract the headers of all the packets that traverse them, but only a sample. The results in this paper suggest that nonlinear support vector machines are highly successful and outperform recent approaches like [12.
\end{abstract}

Keywords: support vector machines, detection, p2p.

\section{Introduction}

In this paper, we apply nonlinear support vector machines 34 to detect peer-topeer (p2p) traffic in high-performance routers with packet sampling. User behavior in research networks has changed radically with the advent of $\mathrm{p} 2 \mathrm{p}$ multimedia file transfers: many users take advantage of the huge bandwidth to exchange movies and the like. This behavior may have a deep impact on research network utilization. We have identified similar concerns in other research networks (http://security.uchicago.edu/peer-to-peer/nofileshare.shtml).

The increasing usage of p2p software in the last three years has raised the need of $\mathrm{p} 2 \mathrm{p}$ detection tools. There are some related initiatives. Among them, in [5], the authors describe a methodology to identify p2p flows at the transport layer, based on connection patterns of $\mathrm{p} 2 \mathrm{p}$ flows, regardless of packet payloads. The authors point that these patterns are more difficult to conceal than explicit flow-conveyed information. In [6], the authors propose to detect p2p traffic by identifying protocol-dependent signatures (key strings) in TCP packet payloads. This is unfeasible in high-performance routers that sample packet headers. However, it may be valid for low-to-medium performance routers. In [1], the authors present a Bayesian classifier of protocols. Although the mean classification accuracy (across all application types) is quite good, the results for $\mathrm{p} 2 \mathrm{p}$ traffic are quite poor (an accuracy of $56 \%$ ). 
The MOLDEIP p2p detection tool we have developed fulfils the following goals: $(i)$ it is independent from router performance, $(i i)$ it is transparent to the users and (iii) it works with sampled packet headers 1 . It does not consider individual flows but the average activity of individual IP addresses. Consequently, there is no short-term technological dependence. Recent network activity is taken from CISCO netflow files, and thus we do not consider packet payloads (unlike the approach in [6]). As in [5], the system is transparent to network users because it does not scan their machines. It works with packet headers, and it does not check them all, but only a sample.

Off-line analysis is feasible because p2p traffic is a nuisance, but it does not disable the network. Thus, a 24-hour decision delay is acceptable.

MOLDEIP employs support vector machines (SVM). Reference [7] also uses SVMs to detect anomalous traffic, but it focuses on intrusion attacks instead of p2p traffic identification. In 2] we demonstrated that linear support vector machine detection of $\mathrm{p} 2 \mathrm{p}$ traffic in high-performance routers with packet sampling is highly successful and outperforms recent approaches like [1]. In this paper we evaluate nonlinear support vector machines for the same purpose.

Specifically, we consider the problem of constructing SVM classifiers based on a given classification of $m$ training vectors (points) in the $n$-dimensional space $\mathbb{R}^{n}$, represented by the $m \times n$ matrix $D$, given the membership of each IP point $D_{i}, i=1, \ldots, m$ in one of two classes - "innocent" or "guilty" -. Each point $D_{i} \in \mathbb{R}^{n}$ is a vector representing an IP address within the Galician RECETGA network, whose components codify the behavior of that IP address.

For this problem, in [2] we followed the linear programming model in [10]:

$$
\begin{aligned}
& \min _{w, z, \gamma, y} e^{\prime} y+e^{\prime} z \\
& \text { such that } C(D w-e \gamma)+\frac{1}{\alpha} y \geq e \\
& \quad-z \leq w \leq z, y \geq 0, z \geq 0,
\end{aligned}
$$

where $w$ is a vector of separator coefficients, $y$ is a vector of slack variables, $\gamma$ is an offset, $\alpha$ is an error penalty, $C$ is a $m \times m$ diagonal matrix with plus ones or minus ones depending on the class of the points represented by $D$ rows, and $e$ stands for a vector of ones of appropriate dimension. This model allows us to employ state-of-the art linear programming solvers. If we compare (1) with the original quadratic model in [3], we see that it tries to minimize the 1-norm of the $w$ parameters instead of their 2-norm (the 2-norm maximizes the margin $\frac{2}{w^{\prime} w}$ between the bounding planes $x^{\prime} w=\gamma \pm 1$ in the standard formulation).

We briefly comment our notation: Capital Latin letters are sets or matrices depending on the context. Lower case Latin letters denote vectors in $\mathbb{R}^{n}$, except for the range $i, \ldots, q$ that denotes integers. Lower case Greek letters are real scalars. Subindices are different components, i.e., $x_{i}$ is the $i$-th component of the $n$-component vector $x$ and $a^{\prime} b$ is the inner product $\sum_{i=1}^{n} a_{i} b_{i}$. For any vector, a $K$ subindex denotes a subvector whose components have indices belonging to the set $K$. For any matrix $B, B_{i}$ is its $i$-th row, and $B_{K}$ is the submatrix composed of all $B_{i}$ such that $i \in K$. For any entity, a superindex is an iteration index.

\footnotetext{
${ }^{1}$ MOLDEIP project, Xunta de Galicia grant PGIDIT03TIC00101CT.
} 
We seek $(i)$ a high classification accuracy, $(i i)$ few nonzero classifier components (for fast operation and identification of statistically relevant information), (iii) few active constraints at the solution of problem (1). The corresponding set $D_{K}$ of support vectors represents problem (1), i.e. the solution does not change if we drop the remaining vectors. When updating classifiers, it is interesting to add to the next problem as few representatives of previous training datasets as possible -i.e. their support vector sets-.

The rest of this paper is organized as follows: in section 2 we model p2p detection. In section 3 we evaluate detection with full monitoring of packet headers. In section 4 we evaluate detection from sampled packet headers, the main goal of this work. Finally, section 5 concludes the paper.

\section{Problem Modeling}

At the time this paper was written, all RECETGA network traffic of the Vigo Campus traversed both a CISCO 7206 router and a Juniper M10 router. The former produced binary netflow files comprising the headers of all traversing packets (origin and destination IP addresses, port identifiers, protocol identifiers, etc), whereas the latter only extracted the information of $0.1 \%$ packets.

We define a new problem each 24-hour slot, from a netflow data batch compiled in the previous slot. First, we convert the netflow files to ASCII format with Flow-tools (http://www.splintered.net/sw/flow-tools/). The resulting 24hour files occupy $2 \mathrm{~GB}$ for the CISCO 7206 and $50 \mathrm{MB}$ for the Juniper M10. Each line in these files corresponds to a single end-to-end transfer, with the following fields: unix time, number of transferred packets, transfer size in bytes, origin IP address, destination IP address, origin port, destination port and transport protocol (TCP or UDP).

Note that there is no flow nor session information: for a given IP address within RECETGA range, there may be thousands of end-to-end IP transfers that appear in the ASCII file as independent entries. Basically, our preprocessor generates a dataset with a single entry per RECETGA IP address.

Remark 1: The fields in each dataset entry match the current high-level metrics in RECETGA graphic analysis tools.

Previous analyses [1] have identified the block sizes and packet formats of most popular p2p protocols nowadays. However, We do not consider explicit p2p protocol information (which can be concealed or encrypted). Our parameters comprise different aggregation levels (day time, night time, 5-minute time slots and 1-hour time slots), to consider temporal behavior.

Let $x$ be a RECETGA IP address. Its dataset entry has the following fields:

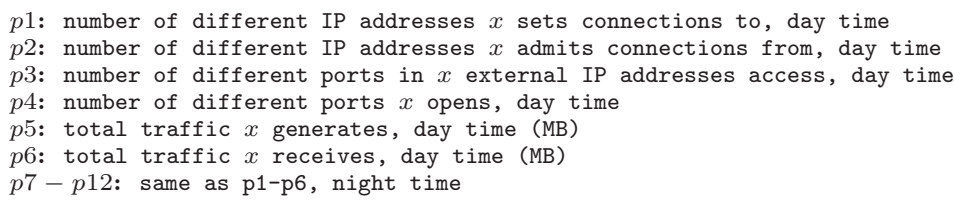




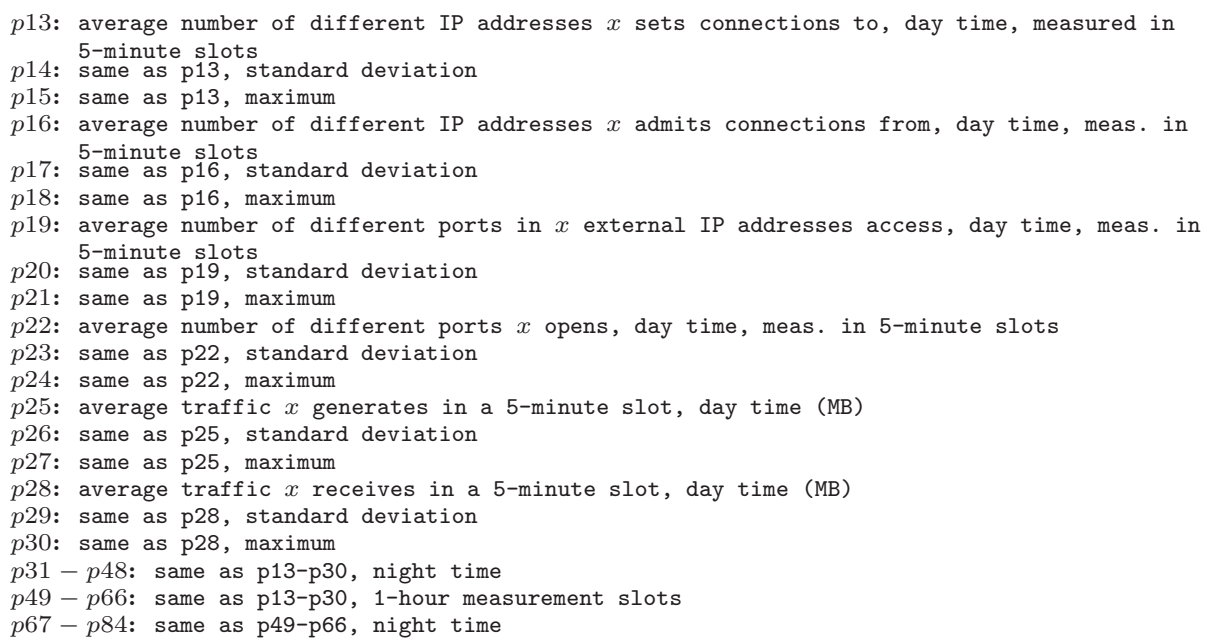

\section{Performance Evaluation, CISCO 7206}

We selected a representative netflow file for the evaluations in this section, corresponding to all RECETGA traffic March 28 2003. This file contained a typical RECETGA behavior without unusual events.

Remark 2: As previously said, at the time this paper was written, all Vigo Campus traffic traversed both the CISCO 7206 and the Juniper M10. Since the CISCO 7206 monitored all traffic, we analyzed its logs $(i)$ to determine the effectiveness of SVMs for p2p off-line detection and $(i i)$ to determine statistically relevant parameter classes. The main goal of this work is $\mathrm{p} 2 \mathrm{p}$ detection from sampled data, as imposed by the Juniper M10. We study that case in section 4.

\subsection{Training and Testing Sets}

First, we applied two filters to the problem:

1. The preprocessor only generated entries for IP addresses belonging to Universidad de Vigo: 6141 IP addresses passed this filter.

2. The preprocessor only generated entries for IPs exchanging over $1000 \mathrm{~KB} /$ day (threshold parameter): 614 IP addresses passed both filters.

The preprocessing times on a Pentium IV (2.4 GHz, 512 MB DDR) are 17.5 hours for $\mathrm{p} 1-\mathrm{p} 12,1.25$ hours for $\mathrm{p} 13-\mathrm{p} 48$ and 7.25 hours for $\mathrm{p} 49-\mathrm{p} 84$. There are three types of parameters: IP parameters, such as $\mathrm{p} 1$ and $\mathrm{p} 2$, port parameters, such as p3 and p4 and traffic parameters, such as p5 and p6.

Once we obtained the dataset, we labeled it as follows to train the classifier:

1. "Guilty" entries: those satisfying any of the following conditions:

(1.1.) using TCP ports of well-known P2P protocols.

(1.2.) large night downloads: $\mathrm{p} 12>100 \times$ threshold 
(1.3.) relatively large uploads: $((\mathrm{p} 5>10 \times$ threshold $)$ AND $(\mathrm{p} 5>\mathrm{p} 6))$ OR $((\mathrm{p} 11>$ $10 \times$ threshold) AND $(\mathrm{p} 11>\mathrm{p} 12))$

2. "Innocent" entries: otherwise.

Remark 3: RECETGA labeling criteria may vary with network regulations.

Remark 4: for further validation of labeling criteria 1-2, we checked the IP addresses in the dataset that established connections with well-known p2p servers. We detected 29 such addresses, which we had correctly labeled as "guilty" ones.

Finally, 456 "innocent" and 158 "guilty" IP addresses resulted. We divided the labeled dataset into one hundred random partitions of training and testing subsets (90\% and $10 \%$ points, respectively). Let $p$ be the percentage of "guilty" points (in this case, $p=0.26$ ). Rather than applying the same factor $1 / \alpha$ to all error variables in (1), we weighted them differently depending on point class: $1 / p \alpha$ for "innocent" points and $1 /(1-p) \alpha$ for "guilty" ones.

\subsection{Parameter Options in SVM Training}

We solved problem (11) on each training subset, and then tested the resulting classifier on the corresponding testing subset. Table 1 shows average results (across the 100 experiments) for parameter options $\mathbf{A}$ and $\mathbf{B}$ below (note: we tuned $\alpha$ in (1) in preliminary trials; $N_{w}$ is the average number of nonzero classifier coefficients at the solution; $N_{s v}$ is the average number of support vectors at the solution; $a_{g}$ and $a_{i}$ are average blind testing accuracies on the testing subsets for "guilty" and "innocent" IP addresses, respectively).

In option $\mathbf{A}$, all parameters participate in the model. In option $\mathbf{B}$, only daytime port parameters participate. This choice is motivated by three facts: first, malicious users may decide to hide behind the bulk of day-time traffic. Second, the parameters that depend on the number of IP addresses will be unfeasible with the advent of the huge IPv6 addressing range. Third, the size of "normal" data transfers keeps growing with the capacity of servers and links. For example, soon all p2p movie exchanges will consist of full DVD contents. Thus, traffic parameters have a strong short-term technological dependence. Note that the labeling criteria in section 3.1 are useless as detection criteria with option B.

Table 1. Average SVM results, options A and B, $\alpha=10$

\begin{tabular}{c|cccc}
\hline \hline Option & $N_{w}$ & $N_{s v}$ & $a_{g}$ & $a_{i}$ \\
\hline $\mathrm{A}$ & 44 & $25 \%$ & $74.1 \%$ & $92.9 \%$ \\
$\mathrm{~B}$ & 11 & $42.4 \%$ & $65.9 \%$ & $93.4 \%$ \\
\hline
\end{tabular}

For option B, the testing accuracy for "guilty" points is low $(\sim 66 \%)$, and the testing accuracy for "innocent" points is high ( 93\%). However, the latter is less important for RECETGA managers than the performance for "guilty" points. As it could be expected, the number of points that support the classifier $\left(N_{s v}\right)$ 
grows when limiting the number of parameters. In any case, even with option $\mathrm{B}$ it is possible to discard $60 \%$ of them. The number of statistically significant parameters is surprisingly low for option B, for a similar performance.

In general, option B seems better than option A. However, in principle, it is not valid due to its low testing accuracy for "guilty" points. In the following subsection we propose a first strategy to correct this problem.

\section{$3.3 \gamma$ Shift}

In 2] we proposed a simple strategy to balance $a_{g}$ and $a_{i}$. "Guilty" points tend to form a "small" cloud at the edge of the "innocent" region in parameter space. Consequently, once the classifier was available, we "tuned" it by shifting $\gamma$ (we refer the reader to [2] for an exact description of this procedure).

The resulting average accuracies of the tuned classifiers are well balanced. For option $\mathrm{A}, a_{g}=90.2 \%$ and $a_{i}=87.1 \%$. For option B, $a_{g}=79 \%$ and $a_{i}=78.7 \%$. We observe that the testing accuracy for "guilty" points grows to $\sim 80 \%$ for option $\mathrm{B}$, which is much better than the results in [1].

For RECETGA managers, a high detection accuracy for "guilty" points is extremely important. In other networks, avoiding false positives may be preferable. Note that $\gamma$-shift is a parameterizable process that can be adjusted accordingly.

\subsection{Faster Preprocessing Stage}

Solving (11) with CPLEX took few seconds in all tests, so preprocessing is dominant in solution time. From the experience of RECETGA managers, port parameters are significant. Thus, in order to decrease preprocessing time, we defined two new parameter options $\mathbf{C}$ and $\mathbf{D}$. Option $\mathrm{C}$ consists of day-time port parameters, aggregation levels of 1 hour and 5 minutes. Option D consists of day-time port parameters, aggregation level of 5 minutes.

Table 2. Average SVM results with $\gamma$ shift, options B-D, $\alpha=10$

\begin{tabular}{c|cccc}
\hline \hline Option & $N_{w}$ & $N_{s v}$ & $a_{g}$ & $a_{i}$ \\
\hline $\mathrm{B}$ & 11 & $42.4 \%$ & $79 \%$ & $78.7 \%$ \\
$\mathrm{C}$ & 10 & $44.3 \%$ & $78.3 \%$ & $78.1 \%$ \\
$\mathrm{D}$ & 6 & $47.2 \%$ & $81.6 \%$ & $81.4 \%$ \\
\hline
\end{tabular}

Table 2 shows the performance of trainer (1) with $\gamma$ shift for these options. We observe that, although the average number of support vectors grows slightly, restricting parameters to 5-minute aggregation also yields the following:

1. Although all parameters in option D are significant, there are only 6 such parameters: p19-p24. In the training scenarios, these parameters appear frequently at the solution with nonzero weights: p19 in 100\% scenarios, p20 in $99 \%$, p21 in $99 \%$, p22 in $87 \%$, p23 in $87 \%$ and p24 in $100 \%$. 
2. An average testing accuracy $a_{g}$ of $81.6 \%$. In other words, only $2-3$ guilty IP addresses escape in each scenario, on average. Apparently, a 5-minute aggregation level captures the evolution of user behavior along the day.

If we preprocess the netflow files with the parameter options so far, the following preprocessing times result: 26 hours for option A, 7.75 hours for option B and 3.25 hours for option C. For option D, we only need $\sim 34$ min of Pentium IV CPU to preprocess 24 hours of RECETGA monitoring data.

\subsection{Nonlinear Kernel Approach}

Although $\gamma$-shift balances classification performance, it has no theoretical support. Therefore, we decided to apply a nonlinear kernel to the best scenario so far -option D- as follows: we extracted a kernel subset of 30 innocent and guilty points $(15+15)$. From the original dataset in option $\mathrm{D}$ we generated a new one by applying the degree 9 polynomial kernel in [8] $K(u, v)=\frac{1}{512}(u /\|u\| \cdot v /\|v\|+1)^{9}$, where $u$ is an original point and $v$ is a point in the kernel subset (both in $\mathbb{R}^{6}$ ). This polynomial kernel has been highly successful in text recognition. Therefore, each original point is mapped into a new one in $\mathbb{R}^{30}$. We only allow a subset of the original points in the kernel subset because this strategy produced good results in 9], for a low increase in computing load.

We ran 100 experiments (reserving $10 \%$ points in each case for testing) with $\alpha=0.01$, and obtained the following results for option $\mathrm{D}: N_{w}=15$ (11 guilty points and 4 innocent points in the kernel subset), $N_{s v}=45.9 \%, a_{g}=80.8 \%$, $a_{i}=83.6 \%$. Thus, the nonlinear support vector machine and the linear one with $\gamma$-shift behave similarly for the CISCO 7206 .

\section{Performance Evaluation, Juniper M10}

In this section we evaluate the impact of packet sampling on $\mathrm{p} 2 \mathrm{p}$ detection accuracy. The Juniper M10 (M10 in the sequel) cannot store all traversing packet headers. In RECETGA, the M10 samples $0.1 \%$ of them. As a result, we relaxed M10 labeling, by setting threshold to $1 \mathrm{~KB} /$ day.

Remark 5: this obvious change is the only design decision to admit sampled data, i.e. we use exactly the same programs and methodologies in section 3 , but we feed them with the sampled headers of the M10.

Table 3 shows SVM detection performance March 28 2003, when RECETGA traffic traversed both the CISCO 7206 and the M10. Full preprocessing was much faster on M10 sampled data: it took less than 30 seconds. So there is no advantage in defining different aggregation levels, in terms of preprocessing time.

Note that the methodologies in section 3 improve on M10 data. If we apply $\gamma$ shift, for less significant port parameters (3 versus 6) and a comparable average number of support vectors, the average testing accuracy for "guilty" points is practically $90 \%$. In all experiments, the three significant parameters in option D with $\gamma$-shift are p19, p21 and p24. 
Table 3. Average SVM results. Option D, Juniper M10 dataset

\begin{tabular}{c|ccccc}
\hline \hline Method & $\alpha$ & $N_{w}$ & $N_{s v}$ & $a_{g}$ & $a_{i}$ \\
\hline Linear w. $\gamma$-shift & 10 & 3 & $50.3 \%$ & $89.3 \%$ & $83.2 \%$ \\
Nonlinear & 0.1 & 6 & $28.3 \%$ & $91.6 \%$ & $90.5 \%$ \\
\hline
\end{tabular}

If we apply the nonlinear kernel in section 3 , only 6 points in the kernel subset are significant at the solution: 2 guilty points and 4 innocent points, on average. The average number of support vectors drops to $28 \%$ and the average testing accuracies (for innocent and guilty points) are well balanced and exceed $90 \%$

\section{Conclusions}

High performance routers cannot monitor all the packet headers, due to their high line rates. As a consequence, techniques such as 5 are unfeasible. Nonlinear support vector machine detection of sampled p2p traffic achieves classification accuracies above $90 \%$. It also outperforms the $\gamma$-shift procedure in [2] in average number of support vectors. The kernel in our experiments was quite small, with as few as six significant points.

\section{References}

1. Moore, A. W. and D. Zuev. Internet Traffic Classification Using Bayesian Analysis Techniques. In Proc. ACM Sigmetrics 2005.

2. González-Castaño, F.J., P.S. Rodríguez-Hernández, R. P. Martínez-Álvarez and A. Gómez-Tato. Support vector machine detection of peer-to-peer traffic in highperformance routers with packet sampling. In Proc. ICANNGA $200 \%$.

3. Vapnik, V. N. The nature of statistical learning theory. Springer, New York, 1995.

4. Cristianini, N. and J. Shawe-Taylor. An introduction to support vector machines. Cambridge University Press, 2000.

5. Karagiannis, T., A. Broido, M. Faloutsos and K.C. Claffy. Transport Layer Identification of P2P Traffic. In Proc. ACM IMC 2004.

6. Sen, S., O. Spatscheck and D. Wang. Accurate, Scalable In-Network Identification of P2P Traffic Using Application Signatures. In Proc. 13th International Conference on World Wide Web, 2004.

7. Tran, Q.A., H. Duan and X. Li. One-Class Support Vector Machine for Anomaly Network Traffic Detection. 2nd Network Research Workshop, 18th APAN, 2004.

8. DeCoste, D. and B. Schoelkopf. Training Invariant Support Vector Machines. Machine Learning, 46:161-190, 2002.

9. González-Castaño, F.J., U. M. García Palomares and R. R. Meyer. Projection Support Vector Machine Generators. Machine Learning, 54-1:33-44, 2004

10. Bradley, P.S., O. L. Mangasarian and D. R. Musicant. Optimization Methods in Massive Datasets. In Handbook of Massive Datasets, J. Abello, P. M. Pardalos and M. G. C. Resende, Editors, Kluwer Publishing, 439-472, 2002.

11. Karagiannis, T., A. Broido, N. Brownlee, K.C. Claffy and M. Faloutsos. Filesharing in the Internet: A characterization of P2P traffic in the backbone. Technical report, Department of Computer Science, University of California, Riverside. http://www.cs.ucr.edu/ tkarag/papers/tech.pdf, 2003. 